\title{
PUBLIC PERCEPTION OF BIODIVERSITY: A LITERATURE REVIEW OF ITS ROLE IN URBAN GREEN SPACES
}

\author{
ARCHANA BELE ${ }^{1} *$ AND UJWALA CHAKRADEO ${ }^{2}$ \\ ${ }^{1}$ Priyadarshini Institute of Architecture and Design Studies, Nagpur, Maharashtra, India- \\ 440019. \\ ${ }^{2}$ Smt. Manoramabai Mundle College of Architecture, Nagpur, Maharashtra, India - \\ 440006. \\ *Corresponding author e-mail: archana.piads@gmail.com
}

Received: $2^{\text {nd }}$ July 2020, Accepted: $18^{\text {th }}$ March 2021

\begin{abstract}
The significance of biodiversity in the survival of human beings and enhancing the urban quality of life is evident from the empirical measurements and qualitative studies carried out across the globe. Despite its importance and value, burgeoning population and growing urbanization are posing a serious threat to biodiversity leading to biodiversity homogenization and ecosystem fragmentation. Moreover, studies reveal that management practices of biodiversity hardly take into account perception, needs, and knowledge of urban residents regarding biodiversity. Urban green spaces have a major role to play in the conservation of urban biodiversity. However, the triangular relationship between biodiversity, urban green spaces, and public perception is still unexplored.

With this aim, the paper attempts to compile, analyze, and synthesize the empirical findings to understand the state-of-the-art knowledge regarding public perception of biodiversity in urban green spaces. The search strategy acquired for the selection of papers resulted in 43 papers from 22 different countries of the world. The paper focuses upon an inclusive definition of urban green spaces, thus encompasses a wide variety of urban and peri-urban green spaces, parks, gardens, and waterfront urban spaces. The analysis of literature pattern reveals a recent increase in studies related to biodiversity perception over the last 10 years. It indicates a strong geographic bias in publications as well. Studies of animals including birds, insects, and reptiles are found scarce compared to plant species. The study could identify potential variables affecting human biodiversity perception which include species literacy, visitation rate, preferences, recreational, health, and restorative benefits, vegetation characteristics, nature connectedness, and conservation support. The paper also proposes a framework for understanding biodiversity perception in urban green spaces that can assist in improving our understanding of the relationship between human interactions and natural environments and framing strategies for urban development, landscape planning, and community health promotions.
\end{abstract}

Keywords: Urban biodiversity; perceptual dimension; species richness; nature connectedness; landscape planning; management policies. 


\section{INTRODUCTION}

An increase in urbanized population is extensively impacting the sustainability of urban systems by affecting their ecological and biophysical components. As a result, the urban population is getting greatly detached from natural environments. Thus, there arises a great demand for urban green space services as these spaces act as essential contributors to human health and quality of life (Maas et. al., 2006; Lee \& Maheswaran, 2011). They have the capacity to enhance resilience and mitigate vulnerability to urbanization (Paul \& Hirani, 2017). Kaplan \& Kaplan (1989) have submitted decades back that exposure to nature is directly related to improved physical (White et. al., 2016), mental (Park et. al., 2010), and psychological health (White et. al., 2017) of the public. Owing to this, the human connection with nature in urbanized areas is of utmost importance, as, by 2050 , about $70 \%$ of the world population is supposed to be living in urban areas (United Nations, 2018), where health challenges are numerous (Dye, 2008).

Moreover, various studies have revealed that people prefer environments with natural elements as compared to built environments (Kaplan \& Kaplan, 1989). This might lead to the supposition that humans positively perceive and prefer environments that are predominantly natural and rich in a diversity of life forms (Williams \& Cary, 2002) i.e., a biodiverse environment.

\section{Urban Biodiversity}

Pickettetal (2016) expresses urban biodiversity as biophysical patterns occurring in the cities. It can also be regarded as an inextricable amalgamation of humans and non-human parts of urban ecosystems. For decades, ecological studies have urged that urbanized environments should be regarded as biodiversity refuges. Hence conservation concerns should be broadened from pristine to urban green areas (Muratet et. al., 2008). But the accelerating pace of urbanization is compelling the cities to be built in the areas of high biodiversity which is alarming the persistence of ecological communities and various species globally (Parrisa et. al., 2018). Biodiversity is under threat due to these challenging conservation issues and hence we need to ensure that the growth of the cities occurs in a biodiversity-friendly way (Miller \& Hobbs, 2002; Marzluff, 2002; Seto et. al., 2012; Dearborn \& Kark, 2010; Shwartz, et. al., 2014b).

In order to conserve biodiversity in urban areas effectively, knowledge from a broad set of disciplines is required (Ahern, 2013). With cooperation from multiple disciplines, urban biodiversity can play a major role in attaining urban sustainability (Jalkanen et. al., 2020). Since, built environment professionals like architects, landscape architects, urban planners, and urban designers directly influence the evolution of the form and fabric of urban environments, they can act as key persons in the endurance and protection of urban biodiversity (Parrisa et. al., 2018).

\section{Urban Green Spaces and Public Perception}

Green spaces have acquired utmost importance in urbanized environments, as they serve as hosts for biodiversity (Cornelis \& Hermy, 2004; Nielsen, et. al., 2013). Apart from aesthetic benefits (Chen et. al., 2009; James et. al., 2009), urban green spaces add to health (Southon et. al., 2018) and recreational benefits, assist in enriching urban cohesion (Germann-Chiari \& Seeland, 2004; Tzoulas et. al., 2007; Ka'zmierczak, 2013), and promote human wellbeing (Arnberger, 2012; Mitchell \& Popham, 2008). Recognition of the synergies amongst these ecological, social, and restorative functions of green space services can assist in better planning of these spaces (Young et. al., 2020). 
It becomes imperative to study the impact of urban green spaces on humans as these spaces provide large scope for studying the human perception of their physical environment particularly, biodiversity. Yet analysis of the human perception of green space and biodiversity within is challenging and thus not much explored (Kothencz \& Blaschke, 2017). This is because of the subjective nature of human perception of the ambient environment where properties of and benefits derived from these spaces differ from person to person (Langemeyer et. al., 2015; Hernández-Morcillo et. al., 2013) and are thus interpreted individually. Moreover, landscape perception is an active process that occurs amid the organisms and their environment (Kaplan \& Kaplan, 1989). Characteristics of a place are greatly influenced and shaped by one's individual perceptions (Tyrväinen et. al., 2007). Greater insight into people's perception of green spaces can assist in better design and management of these spaces and also add to their attractiveness. This might result in greater enjoyment and usage of such spaces and lead people to engage themselves in its conservation initiatives (Shwartz et. al., 2014a). It demands in-depth knowledge of the complex relationship that exists between human well-being, their aesthetic experience, and perceived biodiversity (Hoyle et. al., 2017b). Hence both quantitative, as well as qualitative assessment of public perception of urban green space services, especially biodiversity, is essential for assessment of the urban quality of life.

Unfortunately, urban biodiversity conservation efforts hardly take into account the perceptions, needs, and knowledge of the public. Understanding of lay people's perception of urban green spaces and biodiversity is limited which might add to a crucial knowledge gap in understanding public perceptions and preferences for such spaces. To bridge this gap, interdisciplinary based research is needed urgently.

\section{Public Perception of Biodiversity}

According to Balmford et. al. (2002), people care about the things they know. Bornstein and D'Agostino (1992) and Zajonc (1968) have explained this through the concept of 'mere exposure effect' where familiarity with mere exposure is correlated with positive effects on attitudes and preferences. Based on this, exposure to and knowledge of the people about species can be considered as a good starting point in order to involve the people in biodiversity. But biodiversity is an abstract and challenging concept to convey to the public as it can be interpreted in different ways. (Van \& Wals, 2002).

According to Convention on Biological Diversity (CBD, 1992), biodiversity can be defined as "the variability among living organisms from all sources that include, terrestrial, marine and other aquatic ecosystems along with their ecological complexes incorporating diversity within species, between species, and of ecosystems". Apart from this scientific dimension, it also has social, ethical, and economical dimensions (Gayford, 2000; Wals \& Weelie, 1997). This multi-dimensional character of biodiversity makes it difficult for the general public to grasp, comprehend, and recognize it (Fischer \& Young, 2007; Turner-Erfort, 1997; Lindemann-Matthies \& Bose, 2008).

Though the importance of biodiversity is clear, its role is still unclear i.e., how accurately people perceive it and what are the factors that influence this accuracy is poorly understood (Southon et. al., 2018). This can be investigated through the concept of species literacy amongst the people (Hooykaas et. al., 2019) which is based upon skills of identifying and appreciating the species amongst the people. Various researchers have pointed out that the necessary skills required for identifying and appreciating plants are often lacking particularly amongst the urban public (Clergeau et. al., 2001). In addition to the above, inquiring how experts and the general public conceptualize biodiversity can also help in better planning of 
green spaces and enhancing the role of city residents in the process of planning (Morgan et. al., 2002; Shwartz et. al., 2014a).

Urban green spaces serve as significant recreational resources as well as important habitat for the conservation of flora and fauna in an urban landscape (Zhou \& Chu, 2012) and usually found to possess a higher level of biodiversity as compared to the surrounding urban matrix (Matteson et. al., 2013; Strohbach et. al., 2013).

Most of the research works (e.g., Qiu et. al., 2013) have hypothesized that urban green space users perceive and appreciate biodiversity values while using it for recreation and found that those values are positively related to the preferences. But the knowledge regarding the degree to which the dual purpose of recreation and conservation can be achieved in the same spaces is still not evident. It is also not clear whether the presence of recreational facilities provided in the green spaces conflicts with high biodiversity, i.e., the trade-off between the space available for public amenities and that for natural features. Hence it is imperative to understand the compatibility between recreation and biodiversity and study the correlation between these two components in order to appreciate the benefits derived from such spaces (Wood et. al., 2005; Qiu, 2014).

Qiu et. al. (2013) have pointed out that not much attention has been given to the effect of biodiversity on public preference. One aspect related to this issue is to contemplate whether higher biodiversity, specifically higher vegetation cover attracts or discourages people from visiting the green space. If people prefer to visit the public parks with a lower level of vegetation cover, the benefits derived from this interaction with nature could be constrained to a greater extent.

Apart from this, socio-demographic factors might affect the public perception of biodiversity. These include age, gender, economic status, education level, ethnicity, etc. which might influence the visitation frequency and preference to different types of parks (Jim \& Shan, 2013; Ho et. al., 2005; Zanon et. al., 2013; Lin et. al., 2014; Wende et. al., 2012). For instance, older people might have acquired more knowledge about the biodiverse environment due to more exposure to or more interest in biodiversity (Southon et. al., 2018). Thus, the role of vegetation cover in attracting people from various socio-demographic backgrounds to public parks needs to be studied (Shanahan et. al., 2014).

Though present epidemiological methods testing the interrelation between green spaces and psychological well-being consider all vegetation covers as equal, there might be a possibility that variation in ecological quality might affect the association between green spaces and the health and well-being of the visitors (Wood et. al., 2018). According to Van Den Berg et. al. (2014), experimental laboratory work has proposed that not much relation exists between restorative benefits and different types of natural scenes. Hence it is of utmost importance that the landscape architecture designing actual plant communities should have a better understanding of how people perceive the form, character, and composition of planting shrubs, trees, and plants as most of the research work concerning the human perception of green spaces have focused on designed planting in a much generic sense (Hoyle, 2015).

There is also a lack of evidence about how people with different socio-demographic characteristics experience green space characteristics like vegetation type, its structure, density, and aesthetics predominantly at deliberately designed and managed green spaces (Hoyle, 2019). Moreover, identifying the characteristics of vegetation that are conducive to restoration can assist in designing green spaces that can fetch health and conservation benefits (Young et. al., 2020).

On one hand, biodiversity loss is accelerating day by day due to population growth, unplanned development, and climate change whereas on the other hand, we have begun to 
appreciate the human health benefits that are realized from experiencing nature and biodiversity (Sandifer et. al., 2015). Looking at the growing evidence that human well-being is enhanced by exposure to nature, it becomes vital to understand the relationship between human health and exposure to biodiverse environments as specific qualities of green spaces offering health benefits still remain poorly understood (Dallimer et. al., 2012).

Thus, little is known about various qualities of green spaces that offer benefits to human well-being (Fuller et. al., 2007) particularly with respect to psychological and mental health. One such green space quality that is found to affect human health (psychological and mental) is nature connectedness or one's subjective connection with nature. It is also used as an environmental measure for predicting sustainable attitudes and behaviors (Zelenski \& Nisbet, 2014). The benefits of nature connectedness are rooted in the concept of Biophilia (Wilson, 1984), which focuses on the fundamental intrinsic bond between human beings and nature and based on the postulation that humans have an inherent inclination and affinity with Nature (Grinde \& Patil, 2009). Consequently, it becomes one of the influential aspects of human experience (Kellert, 1997). Thus, measuring one's affective sense of connectedness to nature is also important for understanding the complex relationship between humans and the natural world (Mayer et. al., 2004).

\section{Aim of the Review}

The review aims at compiling, analyzing, and synthesizing empirical findings regarding public perceptions of biodiversity, particularly in urban green spaces. Based on the above studies, the review focuses on addressing, assessing, and drawing broader conclusions about the following questions through scholarly knowledge:

1) To what extent general people acquire knowledge about species diversity/richness (biodiversity)?

2) Whether the presence of recreational facilities conflicts with high biodiversity?

3) Does people's support for biodiversity conservation differ within the population sub-groups?

4) Do socio-demographic factors influence public preference for biodiversity and visitation frequency to green spaces?

5) Do the various types and characteristics of vegetation like its structure, density, aesthetics, etc. affect biodiversity perception and restoration amongst people?

6) Does a correlation exist amongst perception of biodiversity, human health, and people's nature connectedness/relatedness?

\section{METHOD}

The review focuses on empirical data discussing the public perceptions of biodiversity in urban green spaces as well as other spaces visited by them, like, peri-urban protected areas, forested areas, and wetlands thus encompassing an inclusive definition of urban green spaces with an aim of understanding the perception differences amongst these spaces. All these spaces include urban parks, urban gardens, urban meadows, community gardens, urban squares, and streets, peri-urban natural protected areas, forest plantations, woodlands, urban riparian green spaces, wetlands, etc. Papers that reported data regarding urban biodiversity but omitted data about the perception of biodiversity were beyond the scope of this review.

\section{Search Strategy}

The literature search was conducted from February 2020 to April 2020. The search was confined to papers published in the major scientific databases, Scopus and Web of Science 
between 2000 till 2020. The search strategy incorporated either one or a combination of some of the keywords from search terms like 'urban biodiversity, 'public perceptions', 'perception of biodiversity, 'urban green spaces, or 'urban open spaces.' The keywords were searched within the title, abstract, and keywords of the publication, and the search was restricted to peer-reviewed international journal publications that were written in English only. It was realized that though data related to urban biodiversity was enormous, papers relating to perception studies of urban biodiversity were comparatively much lesser. So, the scope was widened beyond the protocol by using snowballing technique thus including the articles that were found relevant while searching for some other empirical data and also those found in the references of the papers obtained from the searches that were focusing on public perceptions of biodiversity and fulfilled the inclusion criteria.

\section{Results of Search Strategy}

Journal wise publications

The search related to perception studies of urban biodiversity generated a total of 42 hits from Scopus and 47 hits from Web of Science. After screening, the articles based on the above search strategy and snowballing technique, 43 potentially eligible papers were retrieved which included 40 research papers, one review paper, and two masters' theses. It was found that the journal publishing the highest number of research papers was Landscape and Urban Planning followed by Urban Forestry \& Urban Greening. In all about 21 journals published the papers revealing that a range of journals shared interest in this topic (Two pieces of research being master's theses are omitted from the count) (Table 1).

Table 1: Journals publishing most research papers on public perceptions of urban biodiversity

\begin{tabular}{|l|c|c|}
\hline Journals containing two or more papers & Number of papers & Percent of papers* \\
\hline Landscape and Urban Planning & 14 & $31 \%$ \\
\hline Urban Forestry \& Urban Greening & 3 & $7 \%$ \\
\hline Land Use Policy & 2 & $4 \%$ \\
\hline Environment and behaviour & 2 & $4 \%$ \\
\hline Biological Conservation & 2 & $4 \%$ \\
\hline Ecosystem Services & 2 & $4 \%$ \\
\hline Journal of Environmental Psychology & 2 & $4 \%$ \\
\hline
\end{tabular}

*Percentage does not add up to $100 \%$ as only journals with 2 or more papers are shown.

\section{Bibliographic Overview of Studies}

Empirical data has been collected through 43 research works on urban green spaces situated in about 22 countries. Prominent geographic unevenness in coverage is evident from Fig. 1 where Europe is showing a large number of studies whereas not many papers were found from African countries and several countries of the Global South. This has also been established by the research work done by Hoyle et. al. (2019). Thus, there exists a knowledge gap in terms of perception studies of urban green spaces in Africa and countries of the Global South. On the contrary, Europe being at the top in perception studies, it could be derived that maximum awareness regarding biodiversity and its perception is amongst the European countries. Interestingly, it was revealed that maximum research work has been carried out in the UK with 14 papers contributing to almost half of the research work done in Europe (Fig. 2) and $33 \%$ of entire papers identified on perceptions of biodiversity from 2000 to 2020 . 
Fig. 1: Continent wise Number of Papers Published

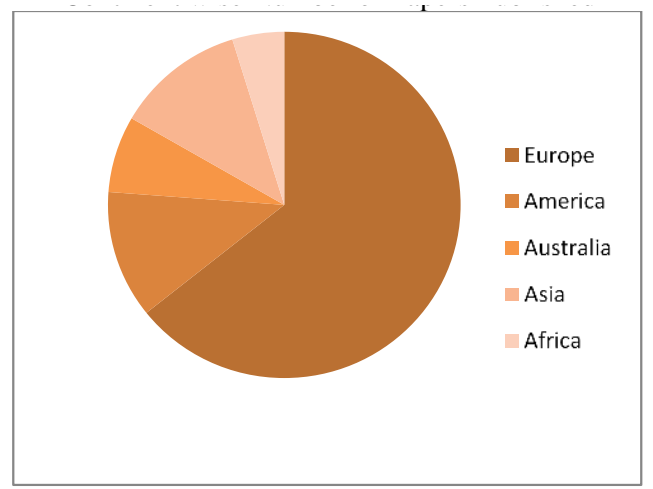

Fig. 2: Country wise Number of Papers Published in Europe

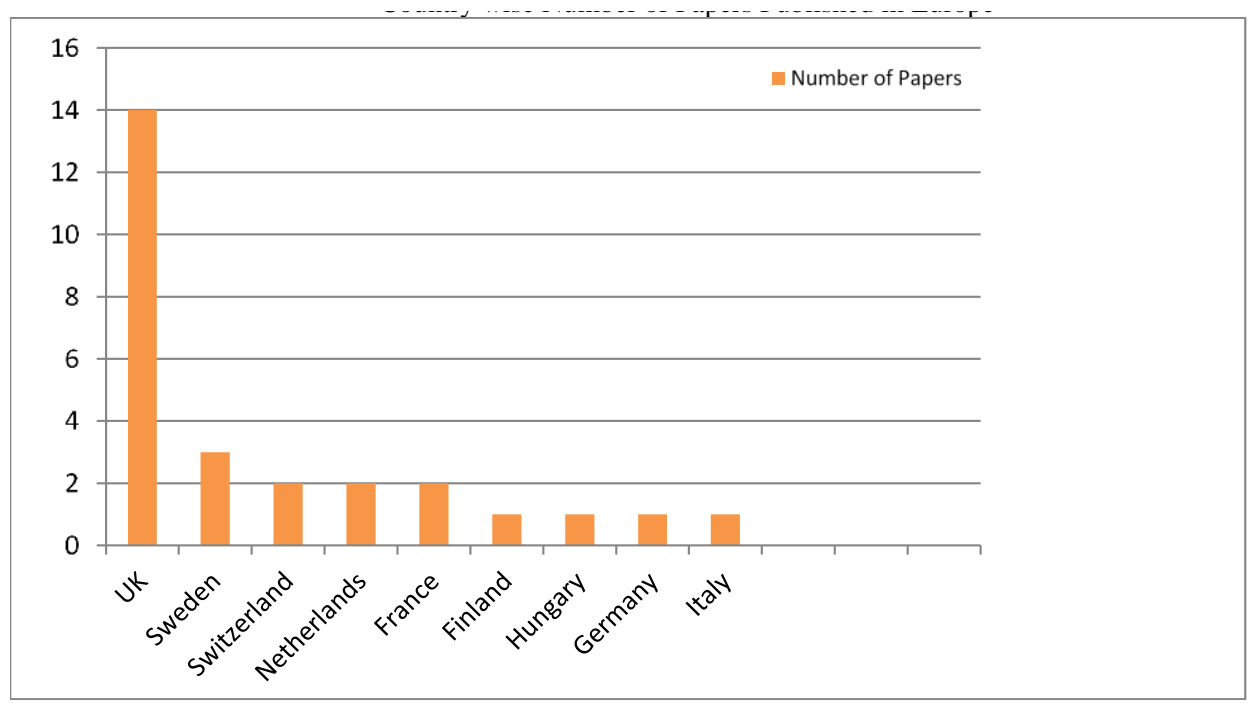

It was also discovered that about $84 \%$ of research work $(n=36)$ has been published from 2010 to 2020 (Fig. 3). This directs towards the fact that the interest in studying urban green spaces and biodiversity perception has gained momentum recently during the last decade only. Recent awareness regarding biodiversity could be related to the enduring global urbanization, growth of urban ecology, and increase in recognition of the significance of biodiversity to human health and wellbeing (Rupprecht et.al., 2015). 
Fig. 3: Number of Papers Published from 2000 to 2020

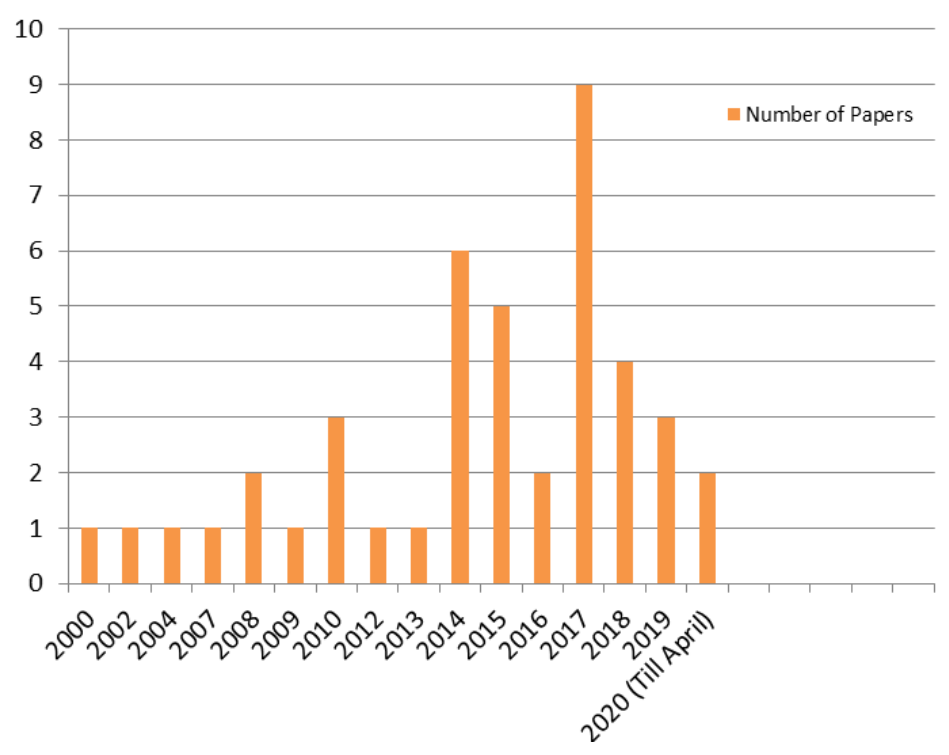

\section{Data Extraction and Synthesis}

Publication patterns have been systematically analyzed and the main findings of all 43 research works along with their implications are discussed after analyzing trends and patterns in literature obtained from Table 2. Results are represented through figures and tabulations to present and synthesize findings from all 43 articles efficiently following similar kinds of presentation and analysis methods used in recent literature reviews (e.g., Rupprecht et. al., 2015). Instead of using quantitative synthesis and meta-analysis in order to determine the overall trends, qualitative synthesis and assessment were deemed appropriate looking at the heterogeneity of the data in terms of the geographical region of study, spaces studied, study methodology, data collection methods, and key findings as applied in other review studies carried out on urban biodiversity (e.g., Nielsen et. al., 2013). 


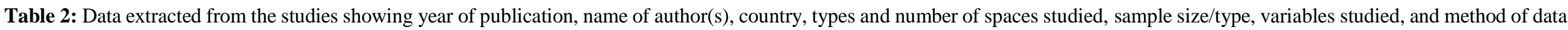
collection used.

\begin{tabular}{|c|c|c|c|c|c|c|c|c|}
\hline Year & Author & Country & $\begin{array}{l}\text { Types of Spaces } \\
\text { Studied }\end{array}$ & $\begin{array}{c}\text { No. of } \\
\text { Spaces } \\
\text { Studied } \\
\end{array}$ & Species Studied & Sample Size/Type & Variables studied & $\begin{array}{l}\text { Data Collection } \\
\text { method }\end{array}$ \\
\hline 2020 & Young et.al. & Switzerland & $\begin{array}{l}\text { domestic and allotment } \\
\text { gardens }\end{array}$ & 301 & Plants & 300 leisure gardeners & Restorative benefits & Questionnaire survey \\
\hline 2020 & Jalkanen et.al. & Finland & Municipal Cities & 4 & $\begin{array}{c}\text { Plants, birds, animals } \\
\text { (mammals), } \\
\text { butterflies, beetles }\end{array}$ & 24 Taxonomic Experts & $\begin{array}{l}\text { Vegetation characteristics } \\
\text { (Spatial prioritization) }\end{array}$ & $\begin{array}{l}\text { Biotope-mapping and } \\
\text { expert opinions. }\end{array}$ \\
\hline 2019 & Hoyle et.al. & UK & $\begin{array}{l}\text { Woodland, shrub and } \\
\text { herbaceous planting }\end{array}$ & 31 & $\begin{array}{l}\text { Plants and animals } \\
\text { (invertebrates) }\end{array}$ & $\begin{array}{c}1411 \text { (survey } \\
\text { questionnaire) and } 34 \text { (in } \\
\text { depth interviews) }\end{array}$ & $\begin{array}{l}\text { Vegetation characteristics, } \\
\text { Nature connectedness }\end{array}$ & Questionnaire survey \\
\hline 2019 & Hooykaas et.al. & Netherlands & - & - & $\begin{array}{c}\text { Birds, animals } \\
\text { (mammals, amphibian } \\
\text { and invertebrates) }\end{array}$ & $\begin{array}{c}4750 \text { (3210 lay people, } \\
602 \text { school children and } \\
938 \text { biodiversity } \\
\text { professionals) }\end{array}$ & $\begin{array}{c}\text { Experts and public } \\
\text { conceptualization of } \\
\text { biodiversity }\end{array}$ & $\begin{array}{l}\text { Questionnaire survey, } \\
\text { species identification } \\
\text { test. }\end{array}$ \\
\hline 2019 & Hassan et.al. & Malaysia & Wetland & 1 & - & 403 & Conservation support & $\begin{array}{l}\text { face-to-face } \\
\text { interviews with urban } \\
\text { and rural households }\end{array}$ \\
\hline 2018 & Campbell-Arvai & Canada & Municipal City & - & - & $\begin{array}{l}18 \text { subject matter experts } \\
\text { and } 28 \text { citizens }\end{array}$ & $\begin{array}{l}\text { Experts and public } \\
\text { conceptualization of } \\
\text { biodiversity }\end{array}$ & $\begin{array}{l}\text { Semi-structured } \\
\text { interviews }\end{array}$ \\
\hline 2018 & Wood et.al. & UK & Urban parks & 12 & $\begin{array}{l}\text { Plants, birds, } \\
\text { butterflies }\end{array}$ & 128 & $\begin{array}{c}\text { Restorative benefits, } \\
\text { Socio-demographic factors }\end{array}$ & $\begin{array}{l}\text { Questionnaire } \\
\text { survey }\end{array}$ \\
\hline 2018 & Southon et.al. & UK & $\begin{array}{l}\text { Experimental perennial } \\
\text { urban meadows }\end{array}$ & 5 & Plants & 240 & $\begin{array}{l}\text { Human health (physical } \\
\text { and mental) Nature } \\
\text { connectedness }\end{array}$ & Questionnaire survey \\
\hline 2018 & Coldwell and Evans & UK & Municipal Cities & 6 & - & 200 respondents & Human health (Mental) & Door-to-door surveys \\
\hline 2017 & Paul and Nagendra & India & City parks & 4 & Plants and animals & 123 & Visitation rate & $\begin{array}{c}\text { Interview, onsite } \\
\text { observation }\end{array}$ \\
\hline 2017 & $\begin{array}{l}\text { Kothencz and } \\
\text { Blaschke }\end{array}$ & Hungary & Urban parks & 5 & Plants & $\begin{array}{l}\text { 125(Field survey), } 130 \\
\text { (Internet survey) }\end{array}$ & Vegetation characteristics & $\begin{array}{l}\text { Questionnaire survey, } \\
\text { GIS }\end{array}$ \\
\hline 2017 & Hoyle et.al. & UK & $\begin{array}{l}\text { Woodland, shrub and } \\
\text { herbaceous planting }\end{array}$ & 31 & Plants & $\begin{array}{c}1411 \text { (survey } \\
\text { questionnaire) and } 34 \text { (in } \\
\text { depth interviews) }\end{array}$ & $\begin{array}{l}\text { Vegetation characteristics } \\
\text { Restorative benefits }\end{array}$ & $\begin{array}{c}\text { Questionnaire survey } \\
\text { and in-depth } \\
\text { interviews }\end{array}$ \\
\hline
\end{tabular}


Bele A., Chakradeo U.: Public Perception of Biodiversity: A Literature Review of its Role in Urban Green Spaces

\begin{tabular}{|c|c|c|c|c|c|c|c|c|}
\hline Year & Author & Country & $\begin{array}{l}\text { Types of Spaces } \\
\text { Studied }\end{array}$ & $\begin{array}{c}\text { No. of } \\
\text { Spaces } \\
\text { Studied }\end{array}$ & Species Studied & Sample Size/Type & Variables studied & $\begin{array}{l}\text { Data Collection } \\
\text { method }\end{array}$ \\
\hline 2017 & Hoyle et.al. & UK & $\begin{array}{l}\text { Woodland, shrub and } \\
\text { herbaceous planting }\end{array}$ & 31 & Plants & $\begin{array}{c}1411 \text { (survey } \\
\text { questionnaire) and } 34 \text { (in } \\
\text { depth interviews) }\end{array}$ & Vegetation characteristics & $\begin{array}{c}\text { Questionnaire survey } \\
\text { and in-depth } \\
\text { interviews }\end{array}$ \\
\hline 2017 & Southon et.al. & UK & $\begin{array}{c}\text { Perennial meadow } \\
\text { creation (experimental } \\
\text { sites and control sites) }\end{array}$ & 10 & Plants & 420 & Preferences (meadows) & $\begin{array}{l}\text { Photo elicitation with } \\
\text { questionnaire survey }\end{array}$ \\
\hline 2017 & Coldwell and Evans & UK & $\begin{array}{l}\text { Small and large urban } \\
\text { areas in pairs }\end{array}$ & 6 & - & 286 & $\begin{array}{l}\text { Species literacy, Visitation } \\
\text { rate, Conservation support }\end{array}$ & $\begin{array}{c}\text { Interview (scales of } \\
\text { pro-environmental } \\
\text { behavior), photo } \\
\text { elicitation }\end{array}$ \\
\hline 2017 & McGinlay et.al. & UK & County & 1 & $\begin{array}{c}\text { Birds, plants } \\
\text { (flowering), butterflies }\end{array}$ & 549 members & $\begin{array}{c}\text { Preferences (charismatic } \\
\text { species) }\end{array}$ & Questionnaire survey \\
\hline 2017 & Hoyle et.al. & UK & $\begin{array}{c}\text { Experimental perennial } \\
\text { meadows }\end{array}$ & 7 & Plants & 8 stakeholder managers & Preference (meadows) & $\begin{array}{c}\text { Semi-structured } \\
\text { interviews with local } \\
\text { authority managers }\end{array}$ \\
\hline 2017 & Palliwoda et.al. & Germany & Recreational parks & 2 & Plants & 15 park users & Vegetation characteristics & $\begin{array}{l}\text { Observation, } \\
\text { interviews }\end{array}$ \\
\hline 2016 & Illiassou et.al. & $\begin{array}{l}\text { Niger (West } \\
\text { Africa) }\end{array}$ & Cities & 2 & Plants and animals & 800 residents & $\begin{array}{l}\text { Conservation support, } \\
\text { Preferences }\end{array}$ & $\begin{array}{c}\text { Ethno biological } \\
\text { survey, questionnaire } \\
\text { survey }\end{array}$ \\
\hline 2016 & Van den Berg et.al. & Netherlands & $\begin{array}{l}\text { Unspectacular natural } \\
\text { and built spaces }\end{array}$ & 40 & Plants & $\begin{array}{l}40 \text { students and university } \\
\text { employees }\end{array}$ & Restorative benefits & Photo elicitation \\
\hline 2015 & Muratet et.al. & France & Urban park & 1 & Plants & $\begin{array}{c}1 \text { botanist and } 100 \text { park } \\
\text { users }\end{array}$ & Vegetation characteristics & $\begin{array}{l}\text { Semi-structured } \\
\text { interview }\end{array}$ \\
\hline 2015 & Hoyle & UK & $\begin{array}{l}\text { Shrubs, woodland, } \\
\text { herbaceous planting }\end{array}$ & 31 & Plants & $\begin{array}{l}1410 \text { Questionnaire } \\
\text { survey, } 34 \text { in depth } \\
\text { interview }\end{array}$ & Vegetation characteristics & $\begin{array}{l}\text { Questionnaire survey } \\
\text { and indepth interview }\end{array}$ \\
\hline 2015 & Sandifer et.al. & - & - & - & - & - & Human health & Literature review \\
\hline 2015 & Carrus et.al. & Italy & $\begin{array}{l}\text { Urban square with green } \\
\text { elements, urban parks, } \\
\text { forest plantation, } \\
\text { peri-urban natural } \\
\text { protected areas }\end{array}$ & 4 & Plants & 569 residents & Restorative benefits & Questionnaire survey \\
\hline
\end{tabular}




\begin{tabular}{|c|c|c|c|c|c|c|c|c|}
\hline Year & Author & Country & $\begin{array}{l}\text { Types of Spaces } \\
\text { Studied }\end{array}$ & $\begin{array}{c}\text { No. of } \\
\text { Spaces } \\
\text { Studied }\end{array}$ & Species Studied & Sample Size/Type & Variables studied & $\begin{array}{l}\text { Data Collection } \\
\text { method }\end{array}$ \\
\hline 2015 & Shanahan et.al. & Australia & Urban parks & 324 & Plants & 670 & $\begin{array}{l}\text { Visitation rate, Nature } \\
\text { connectedness }\end{array}$ & $\begin{array}{c}\text { Questionnaire survey, } \\
\text { (nature relatedness } \\
\text { scale) }\end{array}$ \\
\hline 2014 & Bakhtiari et.al. & Sweden & - & - & - & $\begin{array}{c}51 \text { participants (local } \\
\text { citizens) }\end{array}$ & $\begin{array}{c}\text { Species literacy, } \\
\text { Conservation Support }\end{array}$ & $\begin{array}{l}\text { individual interviews } \\
\text { and group } \\
\text { discussions, thinking } \\
\text { aloud, picture } \\
\text { drawing }\end{array}$ \\
\hline 2014 & Koklukaya et.al. & Turkey & Natural park & 1 & - & $\begin{array}{l}20 \text { prospective science } \\
\text { teachers }\end{array}$ & Biodiversity knowledge & Questionnaire survey \\
\hline 2014 & Qiu & Sweden & $\begin{array}{l}\text { Transect from the } \\
\text { centre of the city to its } \\
\text { outskirts, urban green } \\
\text { spaces, park }\end{array}$ & 8 & Plants & 121 respondents & $\begin{array}{l}\text { Vegetation characteristics, } \\
\text { Recreational benefits, }\end{array}$ & $\begin{array}{l}\text { Biotope mapping, } \\
\text { questionnaire survey, } \\
\text { Visitor employed } \\
\text { photography }\end{array}$ \\
\hline 2014 & Shwartz et.al. & France & Small public gardens & 14 & $\begin{array}{l}\text { Flowering plants, } \\
\text { birds, butterflies, } \\
\text { insects }\end{array}$ & 1116 garden users & Vegetation characteristics & $\begin{array}{l}\text { Semi-structured } \\
\text { interview }\end{array}$ \\
\hline 2014 & Van den Berg et.al. & UK & $\begin{array}{l}\text { urban street, parkland, } \\
\text { tended woodland, wild } \\
\text { woods }\end{array}$ & 4 & Plants & 102 participants & $\begin{array}{l}\text { Vegetation characteristics, } \\
\text { Restorative benefits }\end{array}$ & Video elicitation \\
\hline 2014 & Zelenski and Nisbet & Canada & - & - & - & $\begin{array}{l}331 \text { students and } 619 \\
\text { community people }\end{array}$ & Nature connectedness & Questionnaire Survey \\
\hline 2013 & Qiu et.al. & Sweden & Recreational park & 1 & Plants & 69 park visitors & $\begin{array}{c}\text { Species literacy, } \\
\text { Vegetation characteristics, } \\
\text { Recreational benefits }\end{array}$ & $\begin{array}{l}\text { Visitor-employed } \\
\text { photography (VEP) }\end{array}$ \\
\hline 2012 & Dallimer et.al. & UK & Riparian green spaces & 34 & $\begin{array}{l}\text { Plants, butterflies, } \\
\text { birds }\end{array}$ & 1108 green space visitors & $\begin{array}{l}\text { Human Health } \\
\text { (psychological) }\end{array}$ & Questionnaire Survey \\
\hline
\end{tabular}




\begin{tabular}{|c|c|c|c|c|c|c|c|c|}
\hline 2010 & Vodouhê et.al. & Benin & National Park & 1 & - & 164 residents & Conservation support & $\begin{array}{c}\text { Interview, } \\
\text { questionnaire survey }\end{array}$ \\
\hline 2010 & Leslie et.al. & Australia & Municipal City & 1 & Plants & 94 residents & Vegetation characteristics & Mail survey, GIS \\
\hline 2010 & Hur et.al. & Ohio & Municipal City & 1 & Plants & 725 residents & Vegetation characteristics & $\begin{array}{l}\text { Questionnaire survey, } \\
\text { GIS }\end{array}$ \\
\hline 2009 & Chen et.al. & China & Flower Garden & 1 & Plants & 178 garden visitors & $\begin{array}{l}\text { Vegetation characteristics, } \\
\text { Recreational benefits }\end{array}$ & $\begin{array}{l}\text { Questionnaire survey } \\
\text { and photo-based } \\
\text { evaluation }\end{array}$ \\
\hline 2008 & Matthies \& Bose & Switzerland & $\begin{array}{l}\text { Park, botanical garden, } \\
\text { natural history } \\
\text { museum }\end{array}$ & 3 & Plants & $\begin{array}{c}161 \text { school pupils, } 110 \\
\text { non-graduates and } 96 \\
\text { graduates }\end{array}$ & $\begin{array}{c}\text { Species literacy, } \\
\text { Socio-demographic factors }\end{array}$ & $\begin{array}{l}\text { In depth interview } \\
\text { and questionnaire } \\
\text { survey }\end{array}$ \\
\hline 2008 & Chang et.al. & Taiwan & $\begin{array}{l}\text { naturalness/ wilderness } \\
\text { scenes }\end{array}$ & - & Plants & $\begin{array}{l}110 \text { laboratory } \\
\text { participants }\end{array}$ & Restorative benefits & Photo elicitation \\
\hline 2007 & Fuller et.al. & UK & Green spaces & 15 & $\begin{array}{l}\text { Plants, Birds, } \\
\text { Butterflies }\end{array}$ & 312 green-space users & $\begin{array}{l}\text { Human health } \\
\text { (Psychological) }\end{array}$ & $\begin{array}{l}\text { Semi-structured } \\
\text { interviews }\end{array}$ \\
\hline 2004 & Mayer et.al. & Ohio & - & - & - & $\begin{array}{c}343 \text { (public place visitors, } \\
\text { Psychology students, } \\
\text { Undergraduate } \\
\text { psychology majors) }\end{array}$ & Nature connectedness & Questionnaire survey \\
\hline 2002 & Williams and Cary & Australia & Woodland and forest & 3 & Plants & $\begin{array}{l}1000 \text { urban and rural } \\
\text { resident }\end{array}$ & $\begin{array}{c}\text { Preference, Conservation } \\
\text { support }\end{array}$ & Photo elicitation \\
\hline 2000 & Armstrong & $\begin{array}{l}\text { United States } \\
\text { of America }\end{array}$ & Community Gardens & 63 & Plants & $\begin{array}{l}20 \text { community garden } \\
\text { program coordinators }\end{array}$ & Human health & Telephone interview \\
\hline
\end{tabular}




\section{RESULT}

In all about 1009 urban spaces were studied in the papers with 17 types of urban spaces including urban parks and gardens, urban squares with green elements, wetlands and riparian public green spaces, peri-urban natural protected areas, and woodlands, etc. thus encompassing an extensive category of urban spaces. Out of the total 43 research works, 23 $(54 \%)$ were focusing exclusively on plant species whereas only $1(2 \%)$ on animal species. Nine works $(21 \%)$ were found to be studying both plants and animal species while the remaining 10 works $(23 \%)$ were concerning about the general biodiversity knowledge and conceptualization amongst the public, their support to conservation, and nature connectedness. This pointed out the fact that compared to plant species $(n=32.75 \%)$, perception studies regarding animal species $(n=10.23 \%)$ were very low which led to a very important knowledge gap. Moreover, out of the total animal species studied $(n=10)$, interest in studying birds, butterflies, and beetle species was found to be greater which was evident from 7 papers $(16 \%)$ that discussed bird species and 6 papers $(14 \%)$ that discussed butterfly/insect/beetle species. Also, places like urban parks, gardens, meadows, and herbaceous plantings, were found to be most popular and extensively studied $(\mathrm{n}=25.58 \%)$ while, riparian public green spaces were least studied $(n=2.5 \%)$.

A wide variety of methods (about 15 types) was implemented for collecting the perception data including observations, surveys, interviews, photo, and video elicitation, visitor employed photography (VEP), group discussions, picture drawings, mapping, Geographical Information System, species identification tests, etc. However, the study revealed that most of the researchers still relied on traditional methods of data collection for studying perceptions like questionnaire survey $(n=22)$ which was used by almost half of the researchers $(51 \%)$, and interviews $(n=9)$ used in $21 \%$ of the papers.

\section{Biodiversity Knowledge}

Answer to the first research question could be obtained through two types of papers, those studying general species literacy amongst the public and those exploring the concept of public perception of biodiversity in comparison to the experts.

\section{Species Literacy amongst the Public}

Four papers $(9 \%)$ were found to be discussing species literacy amongst the general public. Results unfolded that higher species literacy is positively associated with higher visitation frequency and it could be enhanced through less intensely urbanized cities having better access to the countryside (Coldwell \& Evans, 2017). While enquiring about the laypeople's understanding of forest ecosystem attributes, Bakhtiari et.al. (2014) realized that people had an instinctive understanding of the ecological concept like biodiversity and values associated with it. Moreover, research carried out by Qiu et. al. (2013) indicated that people can accurately perceive the differences in biodiversity and their knowledge about biodiversity could be positively related to preference to biodiversity. In contrast with this, Matthies \& Bose (2008) realized that laypeople are unaware of the concept of biodiversity and could not recognize it to a wider extent.

\section{Experts and Public Conceptualization of Biodiversity}

Two research works $(5 \%)$ focused on detecting the differences in the understanding of the term 'biodiversity' between the general public and biodiversity professionals and experts. Research indicated that the literacy rate about native species was low amongst laypeople compared to professionals and increased with the age and level of education and found to be associated with a positive attitude towards nature (Hooykaas et. al., 2019). Another research 
revealed that while citizens were knowledgeable about biodiversity, there existed a gap in understanding about some of the drivers of biodiversity loss compared to subject matter experts, i.e., edge effects, habitat isolation, or the destruction of biodiverse habitats (Campbell-Arvai, 2018).

\section{Recreational Benefits and Biodiversity}

Three papers $(7 \%)$ made an inquiry regarding the perceived recreational benefits of biodiversity. Results indicated that ecological knowledge could have a positive influence on preferences for biodiversity with respect to recreational benefits (Qiu et. al., 2013). Chen et. al. (2009) demonstrated that people have different expectations regarding the recreational benefits of urban green spaces in terms of their auditory, tactile, olfactory, and visual qualities. In interdisciplinary research, Qiu (2014) tried to link biodiversity with recreational benefits by studying trade-offs and synergies between biodiversity conservation and recreational values. The research revealed that people could perceive recreational values in urban green spaces through eight sensory dimensions that were associated with biotopes.

\section{Support to Biodiversity Conservation}

Six papers $(14 \%)$ were found to be discussing people's support for biodiversity conservation. While studying conservation support of urban and rural population subgroups to wetlands, Hassan et. al. (2019) realized that urban people favored wetland conservation and had a positive willingness to pay for its conservation whereas rural people were not much willing to pay for conservation. But both groups showed strong preferences for flood risk reduction. Coldwell \& Evan (2017) asserted that regular visitors of green spaces had higher support for conservation and less urbanized cities could maximize public support for biodiversity conservation. People's perception of biodiversity conservation was also seen to be strongly related to perceived benefits derived from the park (Vodouhê et. al., 2010). Similarly, a positive association was found also to exist between landscape preferences and the protection of natural environments (William \& Cary, 2002). According to Bakhtiari et. al. (2014), people's attitudes towards biodiversity might be rooted in their mental constructs that could assist in targeting conservation management practices.

Thus, public perception played an important role in urban space management and could positively contribute to environmental protection policies and decision making (Illiassou et. al., 2016).

\section{Socio-Demographic Factors, Visitation Rate and Preferences to Biodiversity}

Two papers $(5 \%)$ were found to be studying biodiversity perception of people from various social backgrounds, specifically in terms of their education. The study indicated that graduates were most knowledgeable about biodiversity, whereas non-graduates were least (Matthies \& Bose, 2008). Furthermore, young people, as well as adults, held widely inaccurate ideas about plant species richness. Paper by Koklukaya et. al. (2014) attempted to determine the perception of the prospective science teachers which revealed that the teachers were not much aware regarding biodiversity. It pointed out the need for educating prospective teachers about biodiversity and urged to place biodiversity in the educational programs.

Three papers $(7 \%)$ were identified that were discussing visitation rate while 5 papers (12\%) discussed public preferences. A positive association was seen to exist between visitation rate and biodiversity knowledge (Coldwell \& Evans, 2017). In another research, it was realized that the visitation rate was not reliant on the level of tree cover and remnant vegetation (Shanahan et. al., 2015). While analyzing the correlation between frequency of 
use and distance to green spaces, Paul \& Nagendra (2017) pointed out that the visitation rate was dependent upon socio-demographic factors. Compared to old -people, younger visitors traveled long distances to visit green spaces. Also, male visitors had a higher visitation rate in a week than women as women had to face higher constraints while visiting recreational spaces. The number of daily visitors was found to be decreasing with the increase in distance to the green space.

While studying public preferences for biodiversity, it was displayed that most people preferred plants to animals because of their benefits in the form of shade and fruits (Illiassou et. al., 2016). In one of the studies, Williams \& Cary (2002) found that urban people preferred biodiversity in terms of passive and active recreation like walking whereas rural people valued it for its benefit to their stocks in the form of grass. While assessing general people's preferences for meadow style planting, Southon et. al. (2017) explored that people preferred and appreciated meadows compared to both, herbaceous borders and formal bedding planting as well as mown amenity grasslands. Hoyle et. al. (2017a) studied perennial meadow planting with respect to local authority managers' perceptions and discovered that perennial meadows were valued and perceived as a realistic alternative to amenity mown grass that could help in enhancing local biodiversity if implemented in consultation with the general public. It was also found that people prefer high charismatic species groups due to the greater levels of benefits they derive compared to less charismatic species groups (McGinlay et. al., 2017).

In addition to this, various papers were found to be discussing the influence of socio-demographic factors like age and gender on various aspects related to biodiversity such as species richness, restorative benefits, etc. which are discussed in the respective subheads.

\section{Vegetation Characteristics and Restorative Benefits}

Thirteen papers $(30 \%)$ were identified discussing perceptions regarding various characteristics of vegetation while 5 Papers (12\%) studied restorative benefits of biodiversity whereas two papers $(5 \%)$ were found to be discussing the relationship between restorative benefits and vegetation characteristics.

Out of the three papers that used GIS for measuring objective characteristics of green spaces, one paper by Kothencz \& Blaschke (2017) revealed that no strong links existed between objective measures of the urban parks and related subjective evaluations of the parks by the public. Similarly, while using a normalized vegetation index (NDVI) derived from satellite imagery, Leslie et. al. (2010) discovered a lack of agreement between the perceived and objective measures of greenness. They attributed it to the fact that the perceived measure evaluated "ground-level" greenness that is seen from the participants' eyes, while NDVI assessed the amount of green surface seen from above only. The objective measure involved the quantitative aspect of green elements whereas the perceived measure might involve quality of greenness as well. Hur et. al. (2010) used GIS and Landsat satellite imagery and realized that perceived naturalness increased with an increase in perceived openness and as perceived naturalness increased, satisfaction with the presence of trees also increased.

Talking in terms of individual plant species, Hoyle et. al. (2017c) asserted that the majority of people were positive towards non-native species if those were better adapted to climate change. As observed by Palliwoda et. al. (2017), a considerable proportion of activities were seen to be performed related to individual plant species as compared to other park activities. It was also found that as compared to men, more women interacted with plant species. Muratet et. al. (2015) discovered that park users could recognize cultivated plants promoted by the gardeners while botanists mostly observed spontaneous species. 
Regarding species richness, it was established that though people strongly preferred rich diversity of species for their well-being in the gardens, they often underestimated species richness and no significant correlation existed between observed and estimated species richness (Shwartz et. al., 2014). The estimation of plant species richness was also much lower in the case of park users than the botanists. Species character, planting structure, and flowering were determined to have a significant effect on perceptions of neatness, attractiveness whereas socio-demographic factors, beliefs, and values had a lesser influence on their reactions to the planting (Hoyle, 2015). Moreover, the naturalness of the space was perceived as biodiverse, attractive, and restorative, but not essentially tidy and was found to be related to an individual's educational qualification and gender (Hoyle et. al., 2019).

Regarding preference to various types of vegetation, Chen et. al. (2009) found that environmental aesthetic preference did not vary significantly with age and gender but scenic beauty could provide a relaxing environment for the users. People preferred half-open park compared to complex vegetation indicating negative relation between high biodiversity and preference as well (Qiu et. al., 2013). Besides, urban forests were frequently perceived as rich in species than open green spaces (Qiu, 2014). It was also realized that on-site preference to biodiversity was highly context-specific and was triggered by specific features rather than the overall scenery and character of the setting. Jalkanen et. al. (2020) revealed that spatial prioritization could assist in urban land-use and green infrastructure planning in order to maintain biodiversity.

While addressed the question regarding visual features that trigger restorative responses in people, Van den Berg et. al. (2016) concluded that natural scenes were mostly rated more restorative than built scenes. The positive effect of biodiversity was observed on perceived restorative properties and self-reported benefits derived from urban and peri-urban green spaces (Carrus et. al., 2015). Finding by Wood et. al. (2018) indicated that facilities on the site like cleanliness, amenities present, etc. related positively to restorative benefits but unrelated to age, gender, and ethnic background. A large degree of compatibility was found between psychological measures of restorativeness and physiological responses while viewing images of wildland scenes (Chang et. al., 2008). This was established through the improved scores on the perceived restorativeness scale that corresponded to increased EMG (Electromyography) and EEG (electroencephalogram) readings and lower BVP (Blood Volume Pulse) measurements. These findings proved to be significant to the psychophysiological values and benefits of the wildland-wilderness environments for human restoration. Allotment gardens were also found to be highly restorative amongst the gardeners as compared to domestic gardens. A positive association existed between restoration and the number of plant species whereas garden-related stress among a number of gardeners was found to be negatively related to the restoration (Young et. al., 2020).

While exploring the relationship between restorative benefits and vegetation characteristics, a weak correlation was found to exist between perceived attractiveness of the vegetation and restorative effects (Hoyle et. al., 2017b). Though there was a high aesthetic preference for colorful planting, green planting was also greatly valued whereas planting with natural structure was perceived as more restorative. Respondents also described wild woods to be more arousing than the parkland and tended woodland (Van den Berg et. al., 2014).

\section{Biodiversity, Human Health, and Nature Connectedness/Relatedness}

Four papers $(9 \%)$ were identified discussing nature connectedness and 5 papers $(12 \%)$ discussed human health with respect to biodiversity perception. One paper discussed the relationship between both nature connectedness and people's physical and mental health. 
Hoyle et. al. (2019) found naturalness as biodiverse and was related to an individual's connectedness to nature. People with greater nature connectedness traveled even greater distances for more vegetated parks (Shanahan et. al., 2015). Mayer et. al. (2004) used the connectedness to nature scale (CNS) to measure an individual's experiential connection to nature and found the connection to nature as an important predictor of ecological behavior and subjective well-being. According to them, CNS having good psychometric properties could be regarded as a promising tool for research on the relationship between humans and the natural world. Nature-relatedness could also act as a significant predictor of human happiness and environmental sustainability (Zelenski \& Nisbet, 2014).

While exploring health benefits, strong evidence was found linking biodiversity with the production of ecosystem services, nature exposure, and human health (Sandifer et. al., 2015). Fuller et. al. (2007) asserted that psychological health benefits enhanced with the level of species richness of urban green spaces. On the contrary, a lack of consistent relationship was found by Dallimer et. al. (2012) between the psychological well-being of urban green space visitors and actual species richness but well-being was found to be positively related to the richness that the green space users perceived to be present. Coldwell \& Evans (2018) reported that visits to urban green space and countryside related positively to mental well-being while city size and the intensity of urbanization had negligible influence. Biodiversity knowledge did not moderate the association between human well-being and green space visit rates. In a descriptive study by Armstrong (2000) on community garden programs, it was revealed that the most common reasons for visits to community garden programs were access to fresh foods, enjoying nature, and corresponding health benefits.

A positive relationship was found to exists amongst human health (mental and physical), psychological well-being, and nature connectedness as well as between actual and perceived botanical richness (Southon et. al., 2018).

\section{DISCUSSION}

With an intention to analyze the state of the art of research on perceptions of biodiversity, the review collected, collated, and assessed empirical data on perceptions of biodiversity, particularly in urban green spaces.

Overall, the review findings determine that biodiversity knowledge has a greater influence on preference to biodiversity and support for its conservation. Most of the studies demonstrate that though species literacy rate is low amongst the general public compared to subject matter experts, people value biodiversity and it is found to be positively associated with their preferences. It is also established that regular visitors of the green spaces have higher species identification skills thus establishing a positive association between species literacy and visitation rates. Nevertheless, Fuller et. al. (2007) and Dallimer et. al. (2012) have identified contradictory shreds of evidence related to how correctly the general people can assess biodiversity. Research works demonstrating the correlation between perceived and actual biodiversity (Fuller et. al., 2007; Qiu et. al., 2013) found that people most often underestimate biodiversity (Dallimer et. al., 2012).

The findings also determine that people prefer biodiversity in urban green spaces for its recreational benefits. It is evident that people associate recreational benefits with their sensory perceptions in urban green spaces. Thus, the pressure on urban green spaces to serve both the functions of recreation as well as conservation is much higher as recreational activities are often found to conflict with biodiversity conservation (Young et. al., 2005). Hence it becomes significant for the managers and policymakers of the green space to 
understand the correlation and trade-off between the space utilized for site facilities and amenities and the space utilized for green and natural features.

Many researchers have shown interest in studying public awareness and support for biodiversity conservation, particularly the attitude of the public towards the management of biodiversity. The findings reveal that public support for biodiversity conservation is associated with the benefits people derive from biodiversity. This could largely be ascribed to a lack of ecological knowledge and the misconceptions related to the ecological principles that act as major constraints for biodiversity conservation. Moreover, people's support for biodiversity conservation is also discovered to be strongly associated with their origin and level of education (e.g., Vodouhê et. al., 2010), where urban people are found to be more supportive towards conservation practices compared to the rural population. People's biodiversity perceptions, awareness, and conservation support can contribute positively to environmental policies and decision-making and thus should form the basis for urban planning and management (Illiassou et. al., 2016).

It is also evident from the study that perception and preference regarding biodiversity greatly differ amongst various socio-demographic sub-groups and found to increase with age and educational level. Thus, promotion of biodiversity awareness at the school level through exploratory learning activities as suggested by Matthies \& Bose (2008) are felt to be of utmost importance and should become part of formal education.

The visitation rate is found to be varying amongst population sub-groups, e.g., between young and older people as well as males and females. Visitation frequency is also found to be much higher at larger green spaces which fascinate visitors from farther distances. The studies also reveal that people having a greater degree of nature connectedness prefer a higher level of vegetation cover and tend to visit the urban green spaces more frequently which also conforms to the study done by Lin et. al. (2014). Alike biodiversity conservation support, preference for biodiversity also varies greatly amongst population sub-groups based on the benefits derived. Thus, the review directs that efforts should be made to increase the visitation frequency of public to urban green spaces which can assist in enhancing their biodiversity knowledge and promote interest in green spaces resulting in greater conservation support.

Maximum research work regarding biodiversity perception has been carried out on the quality and characteristics of vegetation more specifically species richness (28\%). Though people mostly underestimate species richness, species characteristics significantly affect biodiversity perception. The research works vis-à-vis how human responses to various types of natural landscapes has increased the likelihood that most natural landscape might not always be the most preferred one. Furthermore, it is found that people find natural ecosystems often less attractive than manicured environments (e.g., Kathryn et. al., 2002). But there are certain contradictory pieces of evidence as well (e.g., Žlender \& Thompson, 2017), where it is discovered that there lies a strong cross-cultural preference for green spaces which are semi-natural as compared to the formal parks. Also, actual botanical richness at meadow style planting is found to be strongly linked with perceived botanical richness indicating that people prefer and appreciate meadow style planting (e.g., Southon et. al., 2017 and Hoyle et. al., 2017a). Researchers (e.g. Palliwoda et. al., 2017) recommended several approaches for enhancing biodiversity that include maintenance of the traditional management of meadows, converting lawns into species-rich meadows, and planting shrubs with edible fruits.

Regarding restorative benefits of biodiversity, the findings demonstrate that people largely find biodiversity as restorative and seek numerous health benefits from urban green spaces including physical, mental, and psychological well-being. Though aesthetic preference is 
found to be unrelated to age and gender, people find scenic beauty and natural planting more restoring and relaxing. Thus, as described by Van den Berg et. al. (2014) restoration in urban public spaces depends not only on individual perceptions but also on the physical characteristics of the setting. Hence, as suggested by Wood et. al. (2018), to gain restorative benefits from nature, there is a need to increase the number of urban green spaces, improve vegetation cover with botanical and floral diversity, and enhance species richness and biological complexity within.

Though people only appreciate benefits that are immediately apparent in a general sense (Montgomery, 2002), the studies indicate that people are not completely disconnected from experiencing biodiversity in urban green spaces and still perceive and appreciate species richness present within. Overall, it is observed that vegetation characteristics like planting structure, flowering, quality, and quantity of vegetation have a greater influence on perceptions of biodiversity.

The results across all the studies related to nature connectedness amongst laypeople show that people's orientation towards nature is more often positively related to their preference to biodiversity and visitation rates to the green spaces. Apart from this, people with higher eco-centricity prefer high species richness and tend to identify species more accurately. Significant associations are also found to exist amongst perceived biodiversity, nature connectedness, and self-estimated mental health and psychological wellbeing. It is not just the psychological health but physiological health is also found to be improved with exposure to biodiversity Thus, cultivating and enhancing nature connectedness can prove effective in increasing human happiness and environmentally sustainable behavior (Zelenski \& Nisbet, 2014).

The above analysis and synthesis of empirical findings ultimately assisted in identifying the attributes of biodiversity in urban green spaces, their potential variables and corresponding public perception indicators. These potential variables are found to be interdependent. In addition, though each biodiversity variable is strongly associated with certain human perception indicators, sometimes these perception indicators are found to be overlapping amongst the biodiversity variables. The study led to the understanding of the interrelationships between these factors that have been demonstrated in the proposed framework for studying biodiversity perceptions (Figure 4). 
Fig. 4: Proposed framework for studying human biodiversity perceptions in urban green spaces

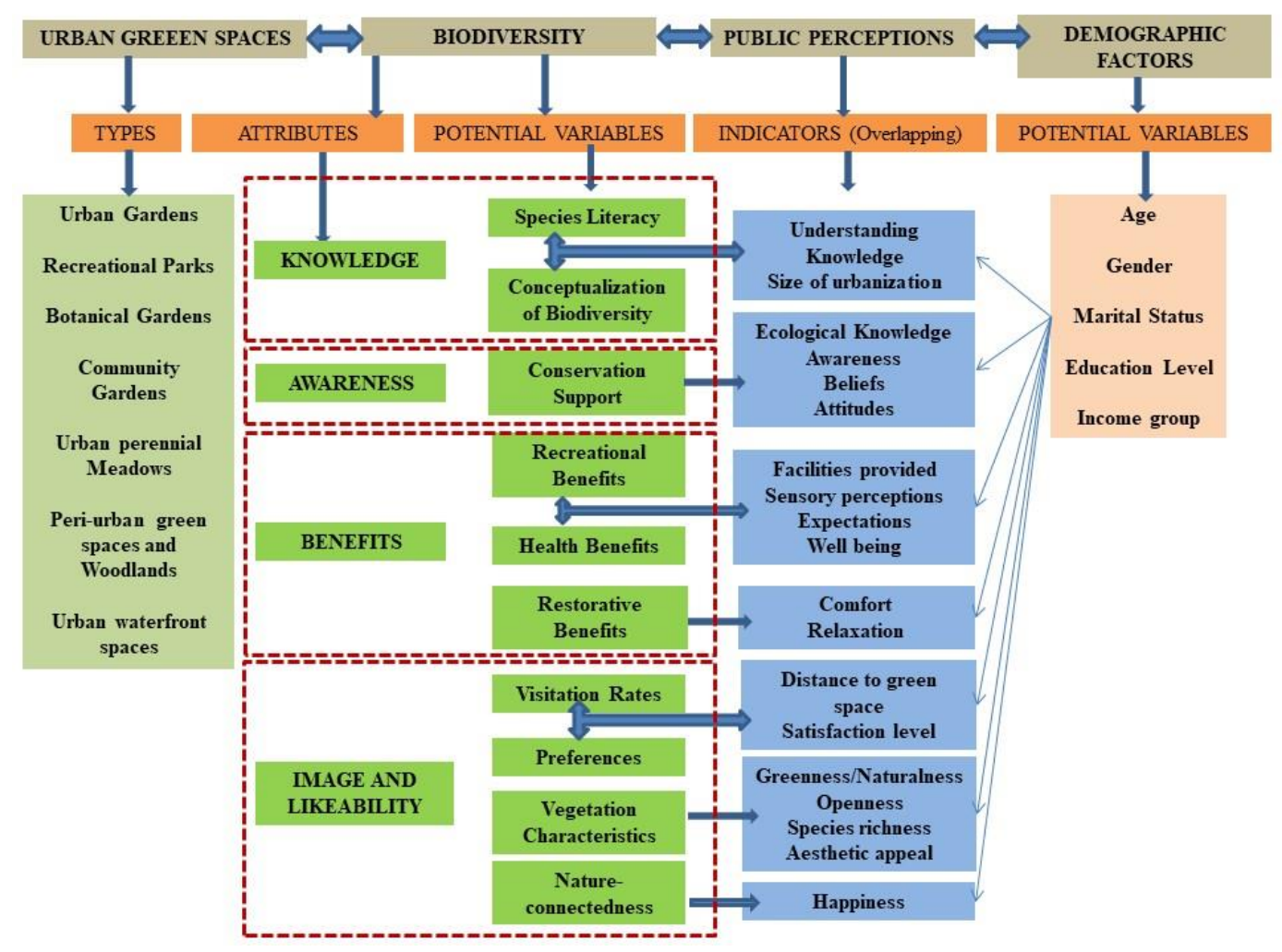

\section{METHODOLOGICAL STRENGTH AND WEAKNESS OF THE RESEARCH}

Looking at the large number and types of spaces studied, it could be derived that the review provides a sound base for generalization of the findings at the local scale. The broader geographical coverage of the studies, which comprises 22 countries located on different continents allows for further generalization of the findings at a global scale. Furthermore, the review collects data from papers that have studied a wide array of urban green spaces and a variety of on-site and off-site data collection methods. At the same time, numerous variables relating to nature and biodiversity have been studied in the papers which further enhance the generality of the review. Most of the studies used observational methods focusing on the analysis of the actual characteristics of biodiversity and species richness and human perceptions rather than relying upon experimental researches in controlled conditions. Thus, the findings across the studies become much more significant for generalization. Having said that, it is also found that no substantial research work has been carried out on the perceptions of biodiversity in countries of Africa and the Global South. The study also indicates that the researchers have been much interested in perceptions studies of plant species and greatly ignored animal species that contribute to a significant limitation of the present state of research on perception studies of biodiversity, more specifically, in urban 
green spaces. Likewise, biodiversity perceptions of waterfront urban spaces particularly riverfronts, lakefronts, etc. also demand greater attention.

The review has some limitations that could be addressed by future researchers. Firstly, the search strategy includes papers published in the English language only as accurate identification and evaluation of research papers in languages other than English were not feasible. Thus, the articles written in languages other than English and other scholarly sources like book chapters are excluded from the review.

Moreover, it is realized that a large amount of scholarly work has been carried out on urban biodiversity and species richness within whereas fewer works are found to be focusing on human perception of urban biodiversity. This results in the inclusion of relatively lesser research papers in this review. This data limitation could be addressed in future systematic review papers by expanding the search and incorporating research works in other languages and from other sources of literature.

Cultural, political, historical, and environmental factors vary greatly across the world. Some more studies exclusively focusing on the effect of these factors on human perception of urban biodiversity might help urban planners and managers to frame and adopt suitable policies for green space development and management.

\section{CONCLUSIONS}

The paper attempts to review the triangular relationship between urban green spaces, biodiversity, and users' perceptions. It is well established from the study that the urban green environment and biodiversity within is greatly appreciated by the users as it offers various benefits in the form of recreation, health, and restoration. At the same time, it also renders unique prospects for the conservation of biodiversity.

However, it could be concluded that there is a necessity for a few more interdisciplinary studies for further investigation of the people-biodiversity connections which would assist in revealing the role of urban biodiversity in people's day-to-day life. It is also felt that to produce stronger evidence, more research work incorporating various other social groups particularly, targeting gender and age differences is sought for. Future studies at other geographical regions and other types of urban green spaces particularly waterfront spaces are also desirable where a negligible contribution has been identified. The research gap is also identified in terms of perceptions of various other taxonomic groups apart from plants like animals, mammals in particular. Future research could also target upon exploring various opportunities of conserving the biodiversity in urban green spaces through public support which could help in the development of advanced ways of conservation and promotion of urban biodiversity.

As such, the growing urbanization has resulted in the present urban societies that are greatly detached from the natural environment which entails higher services from urban green spaces looking at their enormous contribution towards the urban quality of life. Thus, to design and manage urban green infrastructure, there is an urgent need to understand public experiences of nature and how people conceptualize physical, social, psychological, and spiritual services rendered by urban nature.

\section{CONFLICTS OF INTEREST}

The authors declare no potential conflicts of interest with respect to the research, authorship, and/or publication of this article. 


\section{REFERENCES:}

Ahern, J. (2013). Urban landscape sustainability and resilience: The promise and challenges of integrating ecology with urban planning and design. Landscape Ecology, 28, 1203-1212. http://dx.doi.org/10.1007/s10980-012-9799-z.

Arnberger, A. (2012). Urban densification and recreational quality of public urban green spaces-a Viennese case study. Sustainability, 4(4), 703-720.

Armstrong, D. (2000). A survey of community gardens in upstate New York: Implications for health promotion and community development. Health \& Place, 6, 319 \pm 327

Bakhtiari, F., Jacobsen, J.B., Strange, N., Helles, F. (2014). Revealing lay people's perceptions of forest biodiversity value components and their application in valuation method. Global Ecology and Conservation, 1, 27-42, http://dx.doi.org/10.1016 j.gecco.2014.07.003

Balmford, A., Clegg, L., Coulson, T., Taylor, J. (2002). Why conservationists should heed Pokémon. Science, 295 (5564), 2367. https://doi.org/10.1126/science.295.5564. 2367b.

Bornstein, R. F., D'Agostino, P. R. (1992). Stimulus recognition and theme reexposure effect. J. Pers. Soc. Psychol, 63(4), 545-552.https://doi.org/10.1037/0022-3514.63.4.545.

Carrus, G., Scopelliti, M., Lafortezza, R., Colangelo, G., Ferrini, F., Salbitano, F., Agrimi, M., Portoghesi, L., Semenzato, P., Sanesi, G. (2015). Go greener, feel better? The positive effects of biodiversity on the well-being of individuals visiting urban and peri-urban green areas. Landscape and Urban Planning, 134, 221-228, http://dx.doi.org/10.1016/ j.landurbplan.2014.10.022

Campbell-Arvai, V. (2018). Engaging urban nature: improving our understanding of public perceptions of the role of biodiversity in cities. Urban Ecosystems, 22, 409-423. https://doi.org/10.1007/s11252-018-0821-3

Chang, C., Hammitt, W., Chen, P., Machnik, L., Su, W. (2008). Psychophysiological responses and restorative values of natural environments in Taiwan. Landscape and Urban Planning, 85, 79-84, doi:10.1016/j.landurbplan.2007.09.010

Chen, B., Adimo, O. A., Bao, Z. (2009). Assessment of aesthetic quality and multiple functions of urban green space from the users' perspective: the case of Hangzhou Flower Garden, China. Landscape Urban Planning, 93, 76-82.

Clergeau, P., Mennechez, G., Sauvage, A., \& Lemoine, A. (2001). Human percep-tion and appreciation of birds - A motivation for wildlife conservation in urban environments of France. In J. M. Marzluff, R. Bowman, \& R. Donnelly (Eds.), Avian ecology in an urbanizing world (pp. 69-86). Massachusetts: Norwell.

Coldwell, D. F, Evans, K. L. (2017) Contrasting effects of visiting urban green-space and the countryside on biodiversity knowledge and conservation support. PLoS ONE, 12(3): e0174376. https://doi.org/10.1371/journal.pone.0174376

Coldwell, D. F, Evans, K. L. (2018). Visits to urban green-space and the countryside associate with different components of mental well-being and are better predictors than perceived or actual local urbanisation intensity. Landscape and Urban Planning, 175, 114122, https://doi.org/10.1016/j.landurbplan.2018.02.007

Convention on biological diversity. (1992). Rio De Janeiro. Retrieved January 8, 2015, from: http://www. cbd.int/doc/legal/cbd-en.pdf.

Cornelis, J., \& Hermy, M. (2004). Biodiversity relationships in urban and suburban parks in Flanders. Landscape and Urban Planning, 69(4), 385-401. 
Dallimer, M., Irvine, K. N., Skinner, A. M. J., Davies, Z. G., Rouquette, J. R., Maltby, L. L. (2012). Biodiversity and the feel-good factor - Understanding associations between self-reported human well-being and species richness. BioScience, 62, 47-55. http://dx.doi.org/10.1525/bio.2012.62.1.9

Dearborn, D. C., Kark, S. (2010). Motivations for conserving urban biodiversity. Conserv.Biol.24, 432-440. https://doi.org/10.1111/j.1523-1739.2009.01328.x.

Dye, C. (2008). Health and urban living. Science, 319, 766-769. https:// doi.org/10.1126/science.1150198.

Fischer, A., Young, J. C. (2007). Understanding mental constructs of biodiversity: implications for biodiversity management and conservation. Biol. Conserv., 136(2), 271282. https://doi.org/10.1016/j.biocon.2006.11.024.

Fuller, R. A., Irvine, K. N., Devine-Wright, P., Warren, P. H., \& Gaston, K. J. (2007). Psychological benefits of green space increase with biodiversity. Biology Letters, 3, 390394. http://dx.doi.org/10.1098/rsbl.2007.0149

Gayford, C. (2000). Biodiversity education: a teacher's perspective. Environ. Educ. Res. 6 (4), 347-361.

Germann-Chiari, C., Seeland, K. (2004). Are urban green spaces optimally distributed to act as places for social integration? Results of a geographical information system (GIS) approach for urban forestry research. For. Policy Econ., 6, 3-13.

Grinde, B., Patil, G. (2009). Biophilia: Does Visual Contact with Nature Impact on Health and Well-Being. International Journal of Environmental Research and Public Health, 6, 2332-2343; doi:10.3390/ijerph6092332

Hassan, S., Olsen, S., Thorsen, B. (2019). Urban-rural divides in preferences for wetland conservation in Malaysia. Land Use Policy . 84, 226-237, https://doi.org/10.1016/ j.landusepol.2019.03.015

Hernández-Morcillo, M., Plieninger, T., Bieling, C. (2013). An empirical review of cultural ecosystem service indicators. Ecol. Indic. 29, 434-444.

Ho, C. H, Sasidharan, V., Elmendorf, W., Willits, F. K., Graefe, A, Godbey, G. (2005). Gender and ethnic variations in urban park preferences, visitation, and perceived benefits. $J$. Leis. Res., 37(3), 281-306

Hooykaasa, M., Schilthuizena, M., Aten, C., Hemelaar, E., Albers, C., Smeets, I. (2019). Identification skills in biodiversity professionals and lay people: A gap in species literacy. Biological Conservation, 238, 108202, https://doi.org/10.1016/j.biocon.2019.108202

Hoyle, H., Jorgensen, A., Warren, P., Dunnett, N., Evans, K. (2017a). "Not in their front yard" The opportunities and challenges of introducing perennial urban meadows: A local authority stakeholder perspective. Urban Forestry \& Urban Greening, 25, 139-149, http://dx.doi.org/10.1016/j.ufug.2017.05.009

Hoyle, H., Hitchmough, J., Jorgensen, A. (2017b). All about the 'wow factor'? The relationships between aesthetics, restorative effect and perceived biodiversity in designed urban planting. Landscape and Urban Planning, 164, 109-123, http://dx.doi.org/10.1016/j.landurbplan.2017.03.011.

Hoyle, H., Hitchmough, J., Jorgensen, A. (2017c). Attractive, climate-adapted and sustainable? Public perception of non-native planting in the designed urban landscape. Landscape and Urban Planning, 164, 49-63, http://dx.doi.org/10.1016/ j.landurbplan.2017.03.009 
Bele A., Chakradeo U.: Public Perception of Biodiversity: A Literature Review of its Role in Urban Green Spaces

Hoyle, H., Jorgensen, A., Hitchmough, J. (2019). What determines how we see nature? Perceptions of naturalness in designed urban green space. People and Nature, 1, 167-180, doi: 10.1002/pan3.19

Hoyle, H. (2015). Human happiness versus urban biodiversity? Public perception of designed urban planting in a warming climate. Doctoral Thesis, The University of Sheffield, Faculty of Social Sciences, Department of Landscape.

Hur, M., Nasar, J., Chun, B. (2010). Neighborhood satisfaction, physical and perceived naturalness and openness. Journal of Environmental Psychology 30, 52-59, doi:10.1016/j.jenvp.2009.05.005

Illiassou, A., Oumani, A., Abdou, L., Mahamane, A., Saadou, M. (2016). Urban Biodiversity: Perception, Preference, General Awareness, and Threats in Two Cities (Niamey and Maradi) of Niger. Hindawi Publishing Corporation, Urban Studies Research, Volume 2016, Article ID 1469530, http://dx.doi.org/10.1155/2016/1469530

Jalkanen, J., Vierikko, K., Moilanen, A. (2020). Urban Forestry \& Urban Greening, 49, 126586, https://doi.org/10.1016/j.ufug.2020.126586

James, P., Tzoulas, K., Adams, M. D., Barber, A., Box, J., Breuste, J., Elmqvist, T., Frith, M., Gordon, C., Greening, K. L., Handley, J., Haworth, S., Kazmierczak, A. E., Johnston, M., Korpela, K., Moretti, M., Niemelä, J., Pauleit, S., Roe, M. H., Sadler, J. P., Ward, T. C. (2009). Towards an integrated understanding of green space in the European built environment. Urban For. Urban Green., 8, 65-75.

Jim, C. Y, Shan, X. Z. (2013). Socioeconomic effect on perception of urban green spaces in Guangzhou, China. Cities, 31, 123-131.

Kaplan, R. \& Kaplan, S. (1989). The experience of nature: A psychological perspective. New York: Cambridge University Press.

Kazmierczak, A. (2013). The contribution of local parks to neighbourhood social ties. Landscape Urban Planning, 109, 31-44.

Kellert, S. R. (1997). Kinship to mastery: Biophilia in human evolution and development, Washington, DC: Island Press.

Köklükaya, A.N, Demirhan, E., Beşoluk, S. (2014). The Prospective Science Teachers' Perceptions of Biodiversity. Procedia - Social and Behavioral Sciences, 116, 1562 - 1567

Kothencz, G., Blaschke, T. (2017). Urban parks: Visitors' perceptions versus spatial indicators. Land Use Policy, 64, 233-244, http://dx.doi.org/10.1016/j.landusepol. 2017.02.012.

Langemeyer, J., Baró, F., Roebeling, P., Gómez-Baggethun, E. (2015). Contrasting values of cultural ecosystem services in urban areas: the case of park Montjuïc in Barcelona. Ecosyst. Serv., 12, 178-186.

Lee, A.C.K., Maheswaran, R. (2011). The health benefits of urban green spaces: a review of the evidence. J. Public Health, 33, 212-222.

Leslie, E., Sugiyama, T., Ierodiaconou, D., Kremer, P. (2010). Perceived and objectively measured greenness of neighbourhoods: Are they measuring the same thing? Landscape and Urban Planning, 95, 28-33, doi:10.1016/j.landurbplan.2009.11.002

Lin, B. B., Fuller, R. A., Bush, R., Gaston, K. J., Shanahan, D. F. (2014). Opportunity or orientation?: who uses parks and why. PLoS One, 9(1), e87422.

Matthies, L., Bose, E. (2008). How many species are there? Public understanding and awareness of biodiversity in Switzerland. Hum. Ecol., 36 (5), 731-742. 
https://doi.org/10.1007/s10745-008-9194-1.

Maas, J., Verheij, R. A., Groenewegen, P. P., De Vries, S., Spreeuwenberg, P. (2006). Green space, urbanity, and health: how strong is the relation? J. Epidemiol. Community Health, 60, 587-592.

Marzluff, J. M. (2002). Fringe conservation: a call to action. Conserv. Biol., 16, 1175-1176. https://doi.org/10.1046/j.1523-1739.2002.16501.x.

Matteson, K. C., Grace, J. B., Minor, E. S. (2013) Direct and indirect effects of land use on floral resources and flower-visiting insects across an urban landscape. Oikos, 122(5), 682694.

Mayer, F.S., Frantz, C. M. (2004). The connectedness to nature scale: A measure of individuals' feeling in community with nature. Journal of Environmental Psychology, 24, 503-515, doi:10.1016/j.jenvp.2004.10.001

McGinlay, J., Parsons, D., Morris, J., Hubatova, M., Graves, A. (2017). Do charismatic species groups generate more cultural ecosystem service benefits? Ecosystem Services, 27, 15-24, http://dx.doi.org/10.1016/j.ecoser.2017.07.007

Miller, J. R., Hobbs, R. J. (2002). Conservation where people live and work. Conserv.Biol.,16, 330-337.https://doi.org/10.1046/j.1523-1739.2002.00420.x.

Mitchell, R., \& Popham, F. (2008). Effect of exposure to natural environment on health inequalities: an observational population study. The Lancet, 372 (9650), 1655-1660.

Montgomery, C. A. (2002). Ranking the benefits of biodiversity:An exploration of relative values. Journal of Environmental Management, 64(3), 313- 326.

Morgan, M. G., Fischhoff, B., Bostrom, A., Atman, C. J. (2002). Risk communication: a mental models approach. Cambridge University Press, Cambridge.

Muratet, A., Pellegrini, P., Dufour, A., Arrif, T., Chiron, F. (2015). Perception and knowledge of plant diversity among urban park user. Landscape and Urban Planning, 137, 95-106, http://dx.doi.org/10.1016/j.landurbplan.2015.01.003

Muratet, A., Porcher, E., Devictor, V., Arnal, G., Moret, J., Wright, S. (2008). Evaluation of floristic diversity in urban areas as a basis for habitat management. Applied Vegetation Science, 11, 451-460. http://dx.doi.org/10.3170/2008-7-18530

Nielsen, A. B., Van den Bosch, M., Maruthaveeran, S., \& Van den Bosch, C. K. (2013). Species richness in urban parks and its drivers: a review of empirical evidence. Urban Ecosystems, http://dx.doi.org/10.1007/s11252-013-0316-1

Palliwoda, J., Kowarik, I., Von der Lippe, M. (2017). Human-biodiversity interactions in urban parks: The species level matters. Landscape and Urban Planning, 157, 394-406, http://dx.doi.org/10.1016/j.landurbplan.2016.09.003

Park, B. J., Tsunetsugu, Y., Kasetani, T., Kagawa, T., Miyazaki, Y. (2010). The physiological effects of Shinrin-yoku (taking in the forest atmosphere or forest bathing): evidence from field experiments in 24 forests across. Japan. Environ Health Prev Med, 15, $18-26$

Parrisa, K., Amati, M., Bekessy, S., Dagenais, D. (2018). The seven lamps of planning for biodiversity in the city. Cities, DOI: 10.1016/j.cities.2018.06.007

Paul, S., Nagendra, H. (2017). Factors Influencing Perceptions and Use of Urban Nature: Surveys of Park Visitors in Delhi. Land, 6, 27. doi:10.3390/land6020027

Pickett, S.T.A., Cadenasso, M. L., Childers, D. L., Mcdonnell, M. J., Zhou, W. (2016). Evolution and future of urban ecological science: ecology in, of, and for the city. Ecosyst. 
Bele A., Chakradeo U.: Public Perception of Biodiversity: A Literature Review of its Role in Urban Green Spaces

Heal. Sustain. 2. https://doi.org/10.1002/ehs2.1229.

Qiu, L., Lindberg, S., Nielsen, A. (2013). Is biodiversity attractive?- On-site perception of recreational and biodiversity values in urban green space. Landscape and Urban Planning, 119, 136-146, http://dx.doi.org/10.1016/j.landurbplan.2013.07.007.

Qiu, L. (2014). Linking Biodiversity and Recreational Merits of Urban Green Spaces-Methodological Development, Doctoral Thesis, Swedish University of Agricultural Sciences, Alnarp.

Rupprecht, C. D. D., Byrne, J. A., Garden, J. G., Hero, J. M. (2015). Informal urban green space: A trilingual systematic review of its role for biodiversity and trends in the literature, Urban Forestry and Urban Greening (2015), http://dx.doi.org/10.1016/j.ufug.2015.08.009

Sandifer, P. A, Sutton-Grier, A. E., Ward, B. P. (2015). Exploring connections among nature, biodiversity, ecosystem services, and human health and well-being: Opportunities to enhance health and biodiversity conservation. Ecosystem Services, 12, 1-15, http://dx.doi.org/ 10.1016/j.ecoser.2014.12.007

Seto, K. C., Guneralp, B., Hutyra, L. R. (2012). Global forecasts of urban expansion to 2030 and direct impacts on biodiversity and carbon pools. Proc. Natl. Acad. Sci.109,1608316088.https://doi.org/10.1073/pnas.1211658109.

Shanahan, D. F., Lin, B. B., Gaston, K. J., Bush, R., Fulle, R. A. (2015). What is the role of trees and remnant vegetation in attracting people to urban park. Landscape Ecology, 30, 153165, DOI 10.1007/s10980-014-0113-0.

Shwartz, A., Turbé, A., Simon, L., \& Julliard, R. (2014a). Enhancing urban biodiversity and its influence on city-dwellers - An experiment. Biological Conservation, 171, 82-90.

Shwartz, A., Turbé, A., Julliard, R., Simon, L., Prévot, A. C. (2014b). Outstanding challenges for urban conservation research and action. Glob. Environ. Chang., 28, 39-49. https://doi.org/10.1016/j.gloenvcha.2014.06.002.

Southon, G., Jorgensen, A., Dunnett, N., Hoyle, H., Evans, K. (2017). Biodiverse perennial meadows have aesthetic value and increase residents' perceptions of site quality in urban green-space. Landscape and Urban Planning, 158, 105-118, http://dx.doi.org/10.1016/ j.landurbplan.2016.08.003

Southon, G., Jorgensen, A., Dunnett, N., Hoyle, H., Evans, K. (2018). Perceived species-richness in urban green spaces: Cues, accuracy and wellbeing impact. Landscape and Urban Planning, 172, 1-10, https://doi.org/10.1016/j.landurbplan.2017.12.002

Strohbach, M., Haase, D., Kabisch, N. (2009). Birds and the city: urban biodiversity, land use, and socioeconomics. Ecol. Soc. 14(2), 31

Turner-Erfort, G. (1997). Public awareness and perceptions of biodiversity. Transactions of the Illinois State Academy of Science, 90 (3 and 4), 113-121.

Tyrväinen, L., Mäkinen, K., Schipperijn, J. (2007). Tools for mapping social values of urban woodlands and other green areas. Landscape and Urban Planning, 79(1), 5-19.

Tzoulas, K., Korpela, K., Venn, S., Yli-Pelkonen, V., Kazmierczak, A., Niemela, J., James, P. (2007). Promoting ecosystem and human health in urban areas using Green Infrastructure: a literature review. Landscape Urban Planning, 81, 167-178.

United Nations. (2018). Revision of world urbanization prospects. Retrieved December 8, 2018, from: https://www.un.org/development/desa/en/news/population/2018-revision-ofworld-urbanization-prospects.html.

Vodouhê, F. G., Coulibaly, O., Adégbidi, A., Sinsin, B. (2010). Community perception of 
biodiversity conservation within protected areas in Benin. Forest Policy and Economics, 12, 505-512, doi:10.1016/j.forpol.2010.06.008

Van Den Berg, A. E., Jorgensen, A., Wilson, E. R. (2014). Evaluating restoration in urban green spaces: does setting type make a difference? Landscape and Urban Planning, 127, 173-181. doi: 10.1016/j.landurbplan.2014.04.012

Van den Berg, A. E, Joye, Y., Koole, S. (2016). Why viewing nature is more fascinating and restorative than viewing buildings: A closer look at perceived complexity. Urban Forestry \& Urban Greening, 20, 397-401, http://dx.doi.org/10.1016/j.ufug.2016.10.011

Van, W. D., Wals, A. (2002). Making biodiversity meaningful through environmental education. Int. J. Sci. Educ., 24(11), 1143-1156. https://doi.org/10.1080/ 09500690210134839.

Wals, A. Van, W. D. (1997). Environmental education and the learning of ill-defined concepts: the case of biodiversity. S. Afr. J. Environ. Educ., 17, 4-11.

Wende, H. E. W., Zarger, R. K., Mihelcic, J. R. (2012). Accessibility and usability: green space preferences, perceptions, and barriers in a rapidly urbanizing city in Latin America. Landscape and Urban Planning, 107(3), 272-282.

White, M. P., Pahla, S., Wheeler, B. W., Depledge, M. H., \& Fleming, L. E. (2017). Natural environments and subjective wellbeing: Different types of exposure are associated with different aspects of wellbeing. Health \& Place, 45, 77-84. https://doi.org/10.1016 /j.healthplace.2017.03.008

White, M. P., Elliot, L. R., Taylor, T., Wheeler, B. W., Spencer, A., Bone, A., Fleming, L. E. (2016). Recreational physical activity in natural environments and implications for health: A population based cross sectional study in England. Preventive Medicine, 91, 383-388. https:// doi.org/10.1016/j.ypmed.2016.08.023

Williams, K. J. H., Cary, J. (2002). Landscape Preferences, Ecological Quality, and Biodiversity Protection, Environment And Behavior. Vol. 34, No. 2, 257-274. doi10.1177/0013916502034002006.

Wilson, E. O. (1984). Biophilia. Cambridge, MA: Harvard University Press.

Wood, E., Harsant, A., Dallimer, M., Cronin de Chavez, A., McEachan, R. R. C., Hassall, C. (2018). Not All Green Space Is Created Equal: Biodiversity Predicts Psychological Restorative Benefits From Urban Green Space. Frontiers in Psychology, 9, 2320. doi: 10.3389/fpsyg.2018.02320

Young, C., Hofmann, M., Frey, D., Moretti, M., Bauer, N. (2020). Psychological restoration in urban gardens related to garden type, biodiversity and garden-related stress. Landscape and Urban Planning, 198, 103777, https://doi.org/10.1016/j.landurbplan. 2020.103777

Young, J., Watt, A., Nowicki, P., Alard, D., Clitherow, J., Henle, K., Johnson, R., Laczko, E., McCracken, D., Matouch, S., Niemela, J., Richards, C. (2005). Towards sustainable land use: identifying and managing the conflicts between human activities and biodiversity conservation in Europe. Biodiversity and Conservation, 14(7), 1641-1661.

Zajonc, R. B. (1968). Process mapping software. J. Pers. Soc. Psychol, 9(2, Pt.2),1-27.

Zanon, D., Doucouliagos, C., Hall, J., Lockstone-Binney, L. (2013). Constraints to Park Visitation: a meta-analysis of North American studies. Leis. Sci. 35(5), 475-493.

Zelenski, J. M., Nisbet, E. K. (2014). Happiness and Feeling Connected: The Distinct Role of Nature Relatedness. Environment and Behavior, Vol. 46(1), 3-23, doi: 


\subsection{7/0013916512451901}

Zhou, D. Q., Chu, L. M. (2012). How would size, age, human disturbance, and vegetation structure affect bird communities of urban parks in different seasons? J. Ornithol.,153(4), 1101-1112.

Žlender, V., and Thompson, C. (2017). Accessibility and use of peri-urban green space for inner-city dwellers: a comparative study. Landscape and Urban Planning, 165, 193-205. doi: 10.1016/j.landurbplan.2016.06.011 